R E V I S T A

de la

C E P A L

NUMERO 58

ABRIL 1996

SANTIAGO DE CHILE

GERT ROSENTHAL

Director

EUGENIO LAHERA

Secretario Técnico

NACIONES UNIDAS 
Aníbal Pinto Santa Cruz

Gert Rosenthal

Los paradigmas de la política social en América Latina

9

Rolando Franco

Virtudes y limitaciones de los mapas censales de carencias críticas

Rubén Kaztman

Centroamérica: inflación y estabilización en la crisis y poscrisis

Hubert Escaith, Claudia Schatan

El Estado, la empresa y la restauración del paradigma neoclásico

A. Barros de Castro

Globalización y pérdida de autonomía de las autoridades fiscales, bancarias y monetarias

Juan Carlos Lerda

El contexto macroeconómico y la inversión: América Latina

a partir de 1980

Graciela Moguillansky

Los derechos de propiedad y el mercado de la tierra rural en América Latina

Frank Vogelgesang

México: Plan y coyuntura

David Ibarra

Comercio exterior y medio ambiente: experiencias en tres sectores exportadores chilenos

Imme Scholz

El desafío competitivo para la industria brasileña

João Carlos Ferraz, David Kupfer, Lia Hagenauer

Indicadores de la política fiscal: diseño y aplicaciones para Chile

Ricardo Mariner

La CEPAL y la sociología del desarrollo 


\section{Globalización y pérdida de autonomía de las autoridades fiscales, bancarias y monetarias}

\section{Juan Carlos Lerda}

Asesor Regional

en Política Fiscal,

CEPAL
La idea de que la globalización menoscaba la soberanía del moderno Estado-nación es cada día más aceptada en diversos círculos académicos, gubernamentales e internacionales. De hecho, hoy existe una generalizada preocupación por la merma progresiva del margen de maniobra de las autoridades nacionales para decidir asuntos de interés interno con independencia del exterior. Esta percepción se hace extrema en quienes entienden que la globalización ha transformado el Estado-nación en una unidad disfuncional dentro de un mundo sin fronteras. El consenso creado en torno a la tesis señalada es comprensible, dado su carácter general, y sobre todo por la flexibilidad conceptual con que las nociones de globalización y soberanía suelen ser tratadas en diversos círculos profesionales. La primera de ellas se emplea con frecuencia en el ámbito de los medios de comunicación, la cultura, la política y la economía. A su vez, la noción de soberanía es moneda corriente en los campos político y militar, extendiéndose de allí su uso a materias de orden económico. El presente ensayo examina dicha tesis, restringiéndola al campo económico. Aún acotándola así, la idea de que la globalización de la economía reduce el grado de autonomía de las autoridades responsables de la política económica adolece de cierta ambigüedad, ya que la profesión no dispone de una interpretación única o al menos hegemónica de la noción de globalización. Por otra parte, el hecho de que la autonomía no es un concepto originario del campo económico, da lugar a que se lo use con latitud. Para analizar aquí el contenido, alcance y limitaciones de tal tesis, se usan algunos ejemplos tomados del campo de las políticas bancaria, monetaria, cambiaría y fiscal. 


\section{Una caracterización del proceso de}

\section{globalización económica}

Dado que el fenómeno de la globalización viene despertando considerable interés en diversos círculos profesionales, es comprensible que existan varios usos para dicha expresión. A la vez, la regla empírica de que la variedad de opiniones entre economistas es siempre positiva, no encuentra aquí su excepción. Por tal motivo se hace necesario explicar el concepto de ese fenómeno que se ha adoptado en el presente trabajo. ${ }^{1}$

El rasgo que caracteriza la globalización a la que nos estamos refiriendo es un progresivo debilitamiento del grado de territorialidad de las actividades económicas, ya que industrias, sectores o cadenas productivas enteras - sean ellas pertenecientes a la esfera real o a la financiera — pasan a desarrollar sus actividades con creciente independencia de los recursos específicos de cualquier territorio nacional. En tales circunstancias, la localización de las diversas operaciones de una corporación se transforma en una variable de elección para las jerarquías corporativas transnacionales. ${ }^{213}$

La referida independización de las actividades económicas respecto del territorio resulta de causas cuya importancia relativa varía por rama, sector e industria, y también de un país a otro, y que están vinculadas al patrón de progreso técnico, preferencias de los consumidores, organización corporativa y políticas públicas de los gobiernos nacionales. En todo caso, la

\footnotetext{
${ }^{1}$ Respecto del concepto de globalización, véase descripciones más completas en Baumann (1995), Di Filippo (1995), Ornan (1994) y Storper (1995).

${ }^{2}$ Esta caracterización corresponde a Storper (1995). Los 'activos' específicos de un territorio van desde los recursos naturales (depósitos minerales, bosques tropicales, fuerza de trabajo no calificada, etc.) hasta los recursos 'construidos' por la mano del hombre como la dotación de 'analistas simbólicos', los centros financieros, las redes interconectadas y otros servicios computacionales, los aeropuertos, las carreteras, las normas regulatorias favorables (mercados laborales flexibles, etc.) y los tratamientos impositivos preferenciales (incentivos fiscales).

A lo largo del trabajo se usa la distinción entre mercado y jerarquía (vista esta última como la estructura de control y gobierno de una organización), compartiendo la noción expuesta por Williamson de que 'las jerarquías no son otra cosa que la continuación de las relaciones de mercado por otros medios' (Williamson y Winter, 1991).
}

mayor movilidad de los factores (especialmente del capital) propiciada por estas tendencias permite que las jerarquías corporativas elijan y sustituyan territorios dentro de la 'aldea global', sin pérdidas de eficiencia, competitividad y rentabilidad (gráfico 1).

El gráfico 1 permite distinguir analíticamente entre dos fenómenos que habitualmente se dan juntos, pues en mayor o menor medida cada uno de ellos refleja y condiciona la marcha del otro. En primer lugar, un aumento del grado de apertura o internacionalización de la economía nacional (o de un sector de ésta) puede interpretarse como un movimiento en la dirección sur-norte (típicamente desde el cuadrante 3 al 1). En segundo lugar, el fenómeno de globalización de una industria o sector equivale a un movimiento en la dirección este-oeste (típicamente desde el cuadrante 1 al 2). Cuando este último tipo de trayectoria es simultánea en un significativo número de industrias o países, se habla de la globalización de la economía mundial.

Desde una perspectiva más amplia, la globalización es un proceso microeconómico de interacción dinámica entre jerarquías y mercados, que se caracteriza por crecientes interdependencias —entre firmas e

GRÁFICO 1

Corrientes internacionales y territorialidad de las actividades económicas

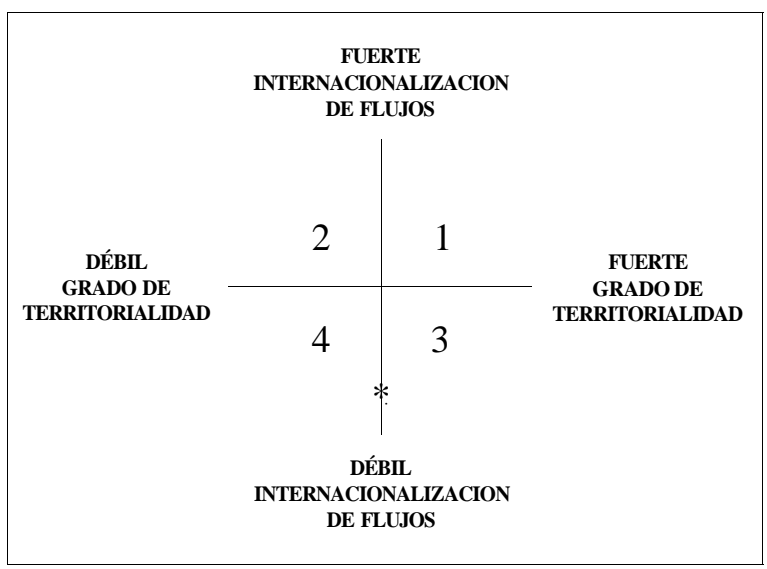

Fuente: Elaboración propia. 
industrias y dentro de ellas- y que vincula organizaciones corporativas y mercados integrados en todo el mundo. Sin embargo, desde el punto de vista de este ensayo, la característica esencial de dicho proceso es la progresiva "desterritorialización" de las actividades económicas. ${ }^{4}$

Por otra parte, el análisis del proceso de globalización se enriquece cuando se lo mira como resultado de complejos procesos evolutivos cuyos antecedentes son más bien remotos. ${ }^{5}$ Así, por ejemplo, la creciente intensificación de flujos - de comercio, inversión extranjera directa, capitales de corto plazo, tecnología- que acompaña, refleja y condiciona el proceso de globalización económica, debe ser interpretada como la aceleración de un proceso histórico cuyas raíces pueden rastrearse en el siglo pasado. ${ }^{6}$

\section{II}

\section{Factores determinantes del proceso de globalización}

El fenómeno de la globalización de la economía mundial se nutre de las tendencias registradas en al menos tres de sus factores determinantes: la tecnología, la organización corporativa y las políticas públicas.

En décadas recientes, dichos factores determinantes han venido interactuando de manera aparentemente cada vez más activa, reforzándose mutuamente y retroalimentándose con renovadas extensiones del propio proceso de globalización. A continuación presentamos una rápida revisión de cada uno de ellos.

\section{Tecnología}

Entre crecer mediante la movilización de recursos (inversión, dinámica demográfica, educación de la fuerza de trabajo, etc.), o haciendo más eficiente su uso (con la incorporación del progreso técnico), hoy en día todo el mundo prefiere este segundo camino. A la vez, dado que aumentar la productividad total de los factores es una tarea que obliga a hacer uso cada día más

\footnotetext{
${ }^{4}$ Ornan (1994) identifica dos usos para el término globalización. El primero hace referencia a un proceso de carácter microeconómico; en este caso el análisis está dirigido, por ejemplo, a temas de competitividad internacional. El segundo uso destaca la noción de globalización como sinónimo de multilateralismo; en este caso el análisis se dirige a temas de política comercial y, de manera especial, a acuerdos sobre el sistema de comercio mundial, como por ejemplo, el Acuerdo General sobre Comercio de Servicios (GATS) y el Acuerdo sobre Aspectos de la Propiedad Intelectual relacionados con el Comercio (ADPIC). Estos últimos - junto con un acuerdo sobre el comercio de bienes (que básicamente actualiza las normas establecidas bajo el GATT) - son los pilares de la nueva Organización Mundial de Comercio (OMC), nacida de la Ronda Uruguay.
}

intensivo de conocimientos, es comprensible que diversas organizaciones -desde empresas transnacionales a gobiernos nacionales - centren crecientemente su atención y esfuerzos en favorecer el desarrollo, la difusión y la adopción de nuevas tecnologías. ${ }^{7}$

Por ello no es de extrañar que durante las últimas dos décadas el mundo haya venido asistiendo a una espectacular ola de innovaciones en áreas claves, en* tre las que destacan la microelectrónica y la informática. Las aplicaciones separadas y conjuntas de innovaciones en estas dos áreas se han reflejado muy favorablemente en los costos de actividades con elevado potencial 'sinérgico' (comunicaciones, almacenaje,

\footnotetext{
${ }^{5}$ Sobre la naturaleza de los procesos evolutivos en economía, véase Nelson y Winter (1982), Nelson (1995) y North (1990); sobre las discontinuidades véase Gerschenkron (1966).

${ }^{6}$ Entre estas raíces destacan i) la función legitimadora de la doctrina del 'libre' comercio heredada de David Ricardo; ii) la reducción de los costos de producción y transporte derivada de las innovaciones que siguieron a la revolución industrial; iii) la consolidación de un sistema de "Estados-nación" anclados en marcos jurídicos compatibles con los derechos de propiedad privada, y los arreglos institucionales que reducían los costos de transacción; iv) la firme determinación, por parte de las potencias coloniales de la época, de implantar el 'libre' comercio y asegurar la apertura de mercados, si fuese necesario, por la fuerza. Ejempo paradigmático de esto último es la llamada 'guerra del opio' (1839-1842) que Inglaterra libró contra China, forzando a este país a abrir sus puertos al comercio de dicha droga y, de paso, transformando Hong Kong en colonia inglesa desde 1842 hasta 1997.

${ }^{7}$ Acerca de la relación entre crecimiento y adopción de tecnologías, véase Parente y Prescott (1991). Otras excelentes contribuciones recientes al análisis económico de la difusión de innovaciones se encuentran en Freeman (1994), Stoneman y Diederen (1994), Metcalfe (1994) y Rosenberg (1994).
} 
transferencia de datos e información, y transportes en general). ${ }^{8}$

Este tipo de avance tecnológico es exactamente lo que las jerarquías corporativas requieren para hacer florecer y consolidar la dimensión microeconómica del proceso de globalización. Dicho avance permite achicar distancias, economizar tiempos, miniaturizar tamaños, reducir pesos, agigantar precisión, aumentar calidad, desagregar o agregar y, en última instancia, posibilita una explosiva expansión de las opciones de que disponen las jerarquías corporativas. Estas pueden así decidir de manera totalmente flexible cómo organizarse, qué y para quién producir, y cómo, cuándo y dónde hacerlo. Vemos así que la progresiva desterritorialización de las actividades económicas debe mucho a las innovaciones en el campo tecnológico.

\section{Organización corporativa}

La fuerte reducción de costos — de producción, comercialización y transacción- propiciada por la incorporación de progreso técnico de tales características abrió las puertas a la adopción de nuevos modelos de organización por parte de las jerarquías corporativas transnacionales (tanto en la esfera real de la economía como en la financiera). El favorable efecto sinérgico de la combinación de innovaciones técnicamente muy avanzadas y sistemas organizacionales caracterizados por su flexibilidad ha contribuido decisivamente a la consolidación del proceso de globalización económica. ${ }^{9}$

Mientras en el pasado reciente la estrategia de las empresas transnacionales frecuentemente consistía en replicar una versión de la firma original a pequeña escala en los países donde se instalaban, el actual modelo de organización tiende a localizar las opera-

\footnotetext{
${ }^{8}$ Al importante impacto económico de la difusión de innovaciones en materia de nuevos materiales, microbiología y biotecnología, debe sumarse el enorme potencial de aplicaciones que vienen mostrando las llamadas 'tecnologías de la información' (TI) resultantes de la combinación del computador, la microelectrónica y las telecomunicaciones en numerosos sectores de la economía. Sobre estas materias véase Milgrom y Roberts (1990), Alcorta (1993) y Rosenberg (1994).

9 Como señala Freeman (1994), cualquier innovación técnica importante involucra cambios en la organización de la producción y de los mercados. A su vez, las innovaciones organizacionales pueden inducir innovaciones técnicas importantes (ejemplos clásicos son la creación de líneas de montaje, la introducción del uso de contenedores, el autoservicio y los supermercados e hipermercados). La actual fase de globalización destaca, entre otros motivos, por la especial e intensa interacción existente entre la tecnología y un sistema de producción integrado a escala mundial, estructurado en torno de una sofisticada división internacional de tareas intrafirma para las principales funciones corporativas. Sobre este tema véase UNCTAD (1993) y Oman (1994)
}

ciones - de producción, abastecimiento, publicidad, asesoramiento jurídico, contabilidad, auditoría, control y manejo de inventarios, investigación y desarrollo de productos y procesos- en diferentes territorios alrededor del mundo.

El nuevo sistema de producción 'flexible' busca ubicar cada función corporativa en el lugar más conveniente, para aprovechar las ventajas comparativas de cada territorio. ${ }^{10}$ Seleccionar la combinación óptima de localizaciones de las distintas funciones u operaciones equivale a construir ventajas porque, entre otras cosas, permite: i) asegurar proveedores de insumos más baratos, de mejor calidad o con mayor proximidad a los centros de producción; ii) situar plantas productoras en lugares estratégicos respecto de los principales centros de venta o consumo de sus productos y iii) usar tecnologías más convenientes, con independencia del origen nacional de las mismas. Todo esto se asemeja a tratar de armar un rompecabezas gigantesco, donde la figura que se busca, así como la localización de las piezas, puede variar según las conveniencias de una estrategia corporativa destinada a asegurar competitividad y rentabilidad para la organización como un todo.

La superioridad económica de este modelo organizacional deriva de su flexibilidad y adaptabilidad a circunstancias generalmente cambiantes y difíciles de anticipar, las que permiten que las jerarquías corporativas mejoren la eficiencia y productividad de sus organizaciones a través de una mayor exposición a la competencia global (esto es, llevándolas a competir con las mejores prácticas de los mejores productores y en los mercados más sofisticados y exigentes, donde sea que se encuentren localizados)."

\footnotetext{
${ }^{10}$ Véase una reciente evaluación de diversas posibles fuentes de ventajas comparativas en Dollar (1993), Learner (1993), Tybout (1993), y Hummels y Levinsohn (1993).

${ }^{11}$ Baily y Gersbach (1995) estudiaron las considerables diferencias en productividad (medida como valor adicionado por hora) que se observan en nueve industrias (automóviles, autopartes, metalmecánica, acero, computadores, productos electrónicos de consumo, alimentación, cerveza, jabones y detergentes) de Estados Unidos, Alemania y Japón, llegando a las siguientes explicaciones: en un primer nivel de análisis, referido al proceso de producción, la mayor parte de las diferencias de productividad pueden ser atribuidas a la manera de organizar las funciones y tareas (innovaciones en el diseño de las manufacturas y la organización del lugar de trabajo) en cada industria y país; factores determinantes tradicionales como la intensidad de uso del capital y la escala de la planta desempeñan un papel menos importante. En un segundo nivel de causalidad, los autores establecen una correlación positiva entre nivel de productividad y grado de exposición de una industria a la competencia global (definida en el sentido indicado en el texto). Esto último refuerza el mensaje derivado de la muy citada punta de 'diamante' de Porter (1991): el secreto de las ventajas competitivas radica en competir (primero con rivales internos y más adelante con los mejores del resto del mundo).
} 


\section{Políticas públicas}

Es evidente que sin la ayuda de políticas públicas compatibles con el funcionamiento de una economía global, la mencionada combinación de tecnología y organización corporativa no habría tenido los efectos hoy conocidos en materia de globalización. Es más, la propia internacionalización o apertura de las economías nacionales — con la escala y características hoy conocidas- es en gran medida obra y gracia de políticas internas compatibles con la intensificación de los movimientos a través de las fronteras. ${ }^{12}$

Ejemplos de políticas públicas que claramente intensificaron las corrientes de comercio durante los últimos cincuenta años fueron los acuerdos gubernamentales para la creación del GATT en 1947. En esa época, los aranceles aduaneros sobre la importación de manufacturas en países industrializados eran de alrededor de $40 \%$, cifra que después de varias rondas de negociaciones (Dillon, Kennedy y Tokio, entre otras) llegó a un promedio de 4\% en la Ronda Uruguay. Esta y muchas otras iniciativas gubernamentales, como las vinculadas a la creación de las Naciones Unidas y sus diversas instituciones especializadas, se insertan dentro del campo de la política externa de los Estadosnación y han dado lugar al ya señalado concepto de globalización entendida como multilateralismo.

Al mismo tiempo, dichas políticas externas también crearon condiciones propicias para el desarrollo de fuerzas globalizadoras en el sentido que aquí más nos interesa. Esto porque los flujos a través de fronteras — cuya intensificación ese 'multilateralismo' se encargó de promover - son el mecanismo a través del cual las organizaciones transnacionales pueden desarrollar y aplicar sus estrategias microeconómicas.

Por otra parte, algunas políticas internas de liberalización y desregulación de mercados -iniciadas en los países industrializados en los años setenta y luego adoptadas por numerosos otros países alrededor del mundo en los años ochenta y primera mitad de los noventa - terminaron de crear las condiciones ideales para que floreciera y luego se consolidara el proceso de globalización económica como fenómeno de carácter microeconómico.

\footnotetext{
${ }^{12}$ Las propias actividades de contrabando - que por cierto deberían contabilizarse como parte del proceso de internacionalización de las actividades económicas - reflejan el efecto de las políticas públicas. Así, por ejemplo, la adopción de una política comercial altamente proteccionista (con arancel medio de importación, digamos, superior a $80 \%$ ), seguramente será percibida como un incentivo (en realidad, un subsidio implícito) para el florecimiento del contrabando.
}

Esas políticas internas fueron formuladas, diseñadas y aplicadas en el contexto de programas de estabilización y ajuste o de reformas estructurales de corte liberalizante, con miras a la búsqueda de mayor eficiencia, flexibilidad, competitividad externa y capacidad productiva de las economías nacionales. Ejemplos conspicuos de ellas son los programas de descentralización fiscal, desregulación financiera, liberalización comercial, privatización o desincorporación de empresas públicas, y las reformas tributarias compatibles con las necesidades lógicas de los anteriores (particularmente con la liberalización del sector externo). El conjunto de políticas asociado con tales programas es bien conocido y responde al nombre de "consenso de Washington". ${ }^{13}$

Ahora bien, el que un mismo conjunto de políticas internas haya sido adoptado en numerosos países de manera casi simultánea, es algo tan poco frecuente que merece una explicación. Por cierto, parece poco creíble la hipótesis de que tan súbita y masiva conversión a una nueva 'sabiduría convencional' pudiera haber resultado de decisiones nacionales totalmente independientes.

Más bien se impone otra interpretación alternativa: la de que los gobiernos nacionales, atrapados entre presiones internas recurrentes y acumulativas de orden distributivo, que no podían ser incorporadas al ya obsoleto modelo de crecimiento hacia adentro, y presiones externas que los afectaban de manera más o menos simultánea, terminaron por adaptarse a las fuerzas que empujaban en pro de la nueva 'sabiduría convencional'. En este proceso, los gobiernos terminaron haciendo suyas las banderas y promesas de esta última. $^{14}$

La creciente movilidad del capital - tangible e intangible, real y financiero-, controlado por agentes económicos transnacionales que operan de manera cada vez más "desterritorializada", fue traduciéndose en exigencias y promesas - concretas y potenciales, explícitas e implícitas, de los mercados y de las propias

\footnotetext{
${ }^{13}$ La expresión 'convergencia universal' ha sido sugerida como alternativa a la muy cuestionada de "consenso de Washington", que acuñó John Williamson. Sobre el origen y evolución de esta terminología ver Williamson, 1993.

${ }^{14}$ Aun cuando la 'versión Banco Mundial' de las causas del 'rnilagro económico del este asiático' sólo recibió difusión masiva con la publicación del informe The East Asian Miracle: Economic Growth and Public Policy (Banco Mundial, 1993), debe recordarse que los planes de estabilización y ajuste de los países que durante los años ochenta firmaron un acuerdo con el FMI y con el propio Banco Mundial contenían los elementos básicos de esa 'sabiduría convencional' (a los que se vinculaban las famosas cláusulas de condicionalidad).
} 
jerarquías corporativas transnacionales-, las que los gobiernos nacionales no pudieron resistir. ${ }^{15}$

De todo lo anterior se concluye que la dirección de la causalidad en la proposición bajo examen - esto es, que la globalización conduce a la pérdida de autonomía - ignora algunas importantes y complejas interacciones de la variable visualizada como determinante (globalización) y del ámbito en el que se produ- ce la eventual pérdida de autonomía (políticas públicas). Por una parte, ignora la importante contribución de la política exterior en pro del multilateralismo para el desarrollo de una economía globalizada. Por otra, dicha visión no hace explícito que la implementación de importantes políticas internas constituye una respuesta racional adaptativa ante presiones externas vinculadas al proceso de globalización.

\section{III}

\section{Formas que puede asumir la perdida de autonomía}

La autonomía, entendida como libertad para regirse o administrarse por sí mismo con independencia de influencias externas, es un concepto nativo del campo político. Así es que para hablar aquí de pérdida de autonomía en el campo económico, debemos importar y luego adaptar aquel concepto a los fines propios de este ensayo.

Esta última tarea no está exenta de riesgos y complejidades. En primer lugar, cabe observar que la esencia misma de los procesos políticos es la negociación. Por ello, habitualmente es imposible distinguir entre lo que un gobierno nacional cede como parte de un acuerdo con los partidos de oposición a fin de obtener alguna ventaja táctica o estratégica (en cuyo caso se habla de una 'hábil negociación política') o cede como resultado de condicionamientos externos vinculados al proceso de globalización (en cuyo caso se hablará de 'nefasta pérdida de autonomía'). Segundo, si negociación política significa ceder en un área con el fin de obtener ventajas en otra a la que se le asigna mayor prioridad, entonces el problema creado por la pérdida de autonomía puede no ser más que el precio necesario para alcanzar otros objetivos. Por

\footnotetext{
${ }^{15}$ Los elementos básicos de esta ni tan nueva 'sabiduría convencional' fueron anticipadamente codificados por Harberger (1984) en un conjunto de 13 reglas para la conducción de la política económica (las que fueron aumentadas a 14 por Fischer, 1987). A su vez, Little, Cooper, Corden y Rajapatirana (1994) propusieron nueve criterios generales con igual propósito. Más recientemente, Harberger (1995) retomó sus recomendaciones, comparándolas con los 10 puntos de convergencia de Williamson. Dos importantes evaluaciones del surgimiento, evolución y eventual caída de la nueva 'sabiduría convencional' se encuentran en Bierksteker (1995) y Krugman (1995).
}

último, es preciso reconocer que la naturaleza del tema no se presta para consideraciones de tipo cardinal. 'Pérdida' de autonomía es una noción de tipo ordinal que deja abiertos espacios donde reinan la indeterminación y la ambigüedad.

En todo caso, el gobierno central del Estado-nación interviene en la economía con cuatro propósitos generales: i) asignar recursos a la provisión de bienes públicos; ii) estabilizar la trayectoria en el tiempo de las variables macroeconómicas (nivel de producción, empleo, precios, moneda, deuda pública y otras); iii) corregir la distribución de ingresos (personal, funcional, regional) y iv) asegurar un ritmo de crecimiento económico adecuado y sustentable. Para ello, habitualmente usa tres canales: i) regulación (fijación de precios y tarifas públicas, determinación de condiciones para la entrada y salida del mercado, localización de actividades, cumplimiento de normas de calidad en la producción y comercialización; ii) política económica (tributaria, arancelaria, de gasto público, monetaria, crediticia, financiera, cambiaria, comercial, industrial) y iii) participación directa como agente productor/distribuidor (empresas de utilidad pública) o financiador (bancos de desarrollo).

Tras tales intervenciones siempre está el supuesto de que las autoridades económicas tienen la capacidad de fijar objetivos y trazar metas compatibles con ellos, seleccionar y utilizar instrumentos eficientes y eficaces, minimizar el costo de sus intervenciones y mantener bajo control el conjunto de restricciones - políticas, económicas y sociales- dentro de las cuales operan sus políticas. Por lo tanto, hablaremos de pérdida de autonomía de las autoridades económicas 
nacionales siempre que el proceso de globalización de la economía mundial afecte de manera adversa el cumplimiento de los anteriores supuestos.

Tal pérdida, a su vez, puede manifestarse de diversas maneras, entre las que destacamos las siguientes:

a) Respecto de los instrumentos

i) Pérdida de instrumentos.

ii) Disminución de la eficiencia o eficacia de ellos.

b) Respecto de las restricciones

Aumento del número, grado de complejidad e importancia estratégica de las restricciones a las que deben ceñirse las autoridades económicas. c) Respecto de los objetivos

i) Aumento del número e importancia de los objetivos de política que dejan de ser opciones viables.

ii) Aumento del costo de insistir en mantener políticas internas alejadas de los mercados y de lo que las jerarquías corporativas transnacionales entienden como 'fundamental'.

iii) Elevación de los estándares para fijar y cumplir metas cuantitativas.

Basándonos en esta clasificación, que tiene sólo fines ilustrativos y está lejos de ser exhaustiva, pasamos ahora a examinar algunos ejemplos vinculados a las políticas monetaria, bancaria, cambiaria y fiscal.

\section{IV}

\section{El caso de las autoridades bancarias y monetarias}

Las referencias al proceso de desterritorialización de las actividades económicas suelen aludir sólo a la actuación de empresas transnacionales que operan en la esfera real de la producción, el comercio y la inversión directa en capitales de riesgo (como hace Dunning, 1993). Sin embargo, el ejemplo más conspicuo de globalización se encuentra en la actuación de los bancos comerciales, los bancos de inversión, las compañías de seguro, las casas de cambio y otros agentes del ramo (incluidas las propias empresas transnacionales en sus relaciones financieras con algunas de aquellas instituciones). Por ello, las actividades y operaciones típicas de los mercados financieros son las que mejor se ajustan a las características del cuadrante 2 del gráfico $1^{16}$

La globalización de los mercados financieros es el prototipo de la interacción sinérgica entre progreso técnico, políticas de desregulación y modelos de organización corporativa. Los avances espectaculares logrados en los sistemas de comunicación y la tecnología de procesamiento, almacenamiento y transmisión

\footnotetext{
${ }^{16}$ La posibilidad de vínculos entre empresas transnacionales y bancos depende del modelo de control corporativo e intermediación financiera vigente en cada país. Véase análisis reciente de las ventajas y limitaciones de los modelos básicos (estadounidense, alemán y japonés) en Porter (1992), Gray (1994) y Prowse (1994).
}

de datos e informaciones han permitido que surjan imaginativas innovaciones financieras caracterizadas por sofisticados 'productos' y complejas técnicas de análisis y gestión. A su vez, la amplia y generalizada desregulación financiera - consistente básicamente en la eliminación de controles sobre movimientos de capitales a través de las fronteras y de límites máximos sobre tasas de interés activas y pasivas- ha promovido mayor competencia interna, movilidad internacional de capitales de corto plazo y creciente integración financiera internacional. ${ }^{17}$

Las cifras citadas por Baumann (1995), GriffithJones y Stallings (1995) y el FMI (1995) respecto a la magnitud y ritmo de variación de algunos flujos inter-

\footnotetext{
${ }^{17}$ El origen de la actual globalización de los mercados financieros puede buscarse en el surgimiento del mercado de eurodólares, a finales de los años cuarenta, cuando la ex-Unión Soviética colocó sus tenencias de dólares en bancos franceses para prevenir una posible congelación de sus cuentas por Estados Unidos. Cuando este último país impuso controles de capital en los sesenta, tanto los bancos como las empresas de ese país 'descubrieron' el mercado de eurodólares como fuente de fondos para sortear los controles de cambios y así financiar sus operaciones. El fin del sistema de Bretton Woods en 1971 y el reciclaje de los petrodólares iniciado en 1973 dieron nueva dimensión a dicho mercado, el que se 'globalizo' de manera definitiva con la amplia desregulación de los mercados financieros iniciada en los años setenta en los países industrializados.
} 
nacionales, tanto en el sector real como en el financiero, indican claramente que la relación cuantitativa que tradicionalmente vinculaba estas dos esferas viene sufriendo una importante discontinuidad. De hecho, puede decirse que los flujos financieros han adquirido vida propia, cruzando fronteras en intensos movimientos especulativos de reiteradas idas y vueltas diarias, con total independencia de la base económica real a la que hasta hace poco se suponían subordinados. La creciente movilidad y desmaterialización de la moneda — hoy reducida a pura información electrónica transmitida de manera instantánea y simultánea por redes de computadores a través del espacio cibernéticohace que las actividades de los mercados financieros se hayan transformado en un paradigma de la desterritorialización de las actividades característica del proceso de deglobalización. ${ }^{18}$

Como es fácil imaginar, toda esta nueva situación viene creando enormes desafíos para las autoridades de los bancos centrales y demás responsables por la conducción de las políticas bancaria, monetaria y cambiaria.

En primer lugar, la extraordinaria ampliación de las interdependencias entre instituciones financieras —nacionales y extranjeras-, posibilitada por la desregulación y las innovaciones que acompañan al proceso de globalización, viene aumentando el riesgo sistêmico. Este último invariablemente se origina a nivel microeconómico con la eventual incapacidad de una institución financiera de cumplir con sus obligaciones, lo que puede arrastrar a otras —en una reacción en cadena - a una situación de insolvencia y, eventualmente, a la quiebra del propio sistema. Como es fácil comprender después del episodio del Banco Barings, una creciente vulnerabilidad externa equivale a una efectiva reducción de la eficiencia y eficacia de los instrumentos de supervisión bancaria.

La mayor fragilidad del sistema financiero deriva de:

i) la creciente opacidad de los estados de cuentas y balances de sus instituciones, lo que se explica por el intenso uso de productos derivados y otros instru-

\footnotetext{
18 O'Brien (1992) acuñó la expresión 'el fin de la geografía' (posiblemente por analogía con 'el fin de la historia' de Fukuyama) como metáfora que resume la noción de desterritorialización de buena parte de las operaciones financieras contemporáneas. Estas últimas, en el decir de mi colega Edgardo Noya, tienen lugar en el 'espacio Borgeano' donde se juntan lo infinito con lo simultáneo de manera instantánea.
}

mentos de extremada complejidad técnica, con riesgos asociados muy difíciles de estimar; ${ }^{19}$

ii) una mayor fragilidad del sistema de pagos interbancários, que ha pasado a ser el eslabón más débil en la cadena de arreglos institucionales destinados a evitar una crisis sistêmica; esto se debe al alza explosiva del valor de los pagos efectuados a través de dichos mecanismos en relación con los recursos netos medios de que disponen los participantes del sistema, lo que significa que una eventual falla de sincronización - por problemas de liquidez de un agente- podría generar una crisis sistêmica de proporciones, ${ }^{20}$

iii) la inestabilidad de los precios de los activos financieros, traducida en mayor volatilidad de corto plazo (como en los mercados de divisas), o, lo que es más serio, en precios desalineados en el mediano plazo (como en los mercados de cambio, propiedades y títulos durante gran parte de los años ochenta en algunos países industrializados). Como es fácil imaginar, cuando estas burbujas explotan, crece la probabilidad de riesgo sistêmico.

$\mathrm{El}$ aumento de ese riesgo sistêmico que resulta de la globalización de los mercados financieros se traduce en potenciales efectos adversos sobre la conducción de las políticas monetaria y fiscal. En el ámbito monetario, una inyección de crédito para recuperar instituciones 'demasiado grandes para quebrar' constituye una amenaza para la trayectoria de los agregados monetarios y las expectativas inflacionarias. En el ámbi-

\footnotetext{
${ }^{19}$ Acerca de la falta de transparencia de los estados de cuentas y balances de las instituciones financieras, el actual Presidente del Instituto Monetario Europeo y hasta hace poco tiempo Gerente General del Banco de Pagos Internacionales manifestó recientemente: "Para decirlo incisivamente, yo desafiaría incluso a un profesional de las finanzas a que tome las cuentas publicadas de algún gran banco internacional de otra institución financiera y haga una evaluación exacta de sus asuntos... Esta falta de transparencia no sólo anula uno de los supuestos claves que la economía libre necesita para efectuar una óptima asignación de recursos, sino que también significa que los participantes tal vez no estén en condiciones de aplicar a las instituciones financieras la disciplina de mercado de facto necesaria para evitar el riesgo de que surjan problemas sistêmicos" (Lamfalussy, 1995. Cita traducida del inglés).

${ }^{20}$ Es sabido que cuando los agentes se retiran de las cajas de compensación bancaria porque anticipan problemas con su funcionamiento, se genera una crisis de liquidez que puede terminar en una crisis sistêmica. Este ejemplo de profecías autocumplibles recibe el nombre de "riesgo Herstatt", en recuerdo de un banco alemán que quebró en 1974 por dicho motivo. Por otra parte, según Crockett - actual Gerente General del Banco de Pagos Internacionales- se estima que en dos días y medio de operación, los sistemas de compensación de pagos interbancários de Suiza y Japón movilizan recursos equivalentes al PIB anual de cada país. En Estados Unidos y Alemania el tiempo requerido varía de tres a cuatro días (Crockett, 1994).
} 
to fiscal, una eventual crisis sistemica puede representar un fuerte aumento de la carga tributaria que se precisa para retomar los equilibrios macroeconomicos.

En segundo lugar, es obvio que a medida que las instituciones financieras internas se tornen más vulnerables, debido a las interdependencias asociadas con la globalización del sistema financiero mundial, las autoridades de los bancos centrales nacionales pierden incentivos para controlar la expansión de los agregados monetarios de manera compatible con la reducción de la tasa de inflación anual. En tal caso, dichas autoridades tendrán como primera prioridad velar por la integridad del sistema financiero, dejando en segundo plano la política antiinflacionaria.

En tercer lugar, la explosión de innovaciones financieras y la volatilidad de los precios de activos como divisas, propiedades, títulos y acciones, tienden a reducir la estabilidad de la relación entre grandes agregados monetarios y el PIB. De esta manera la globalización financiera hace que los mecanismos de transmisión monetaria sean cada vez menos predecibles (con lo cual se reduce la eficiencia y eficacia de la política monetaria).

En cuarto lugar, la existencia de mercados de divisas que operan alrededor del mundo las 24 horas del día, con información puesta en la pantalla de computadores conectados en red, permite la instantánea sustitución de monedas en los portafolios de los inversionistas. Este fenómeno de sustitución monetaria entre países sugiere que el nivel más eficiente para controlar la expansión monetaria puede ser el supranacional. $^{21}$

En quinto lugar, con suficiente movilidad internacional del capital financiero, una política que fije la tasa nominal de cambio y no imponga ningún control a la compraventa de divisas hace endógena la oferta de base monetaria (o sea, se pierde autonomía en el manejo de la política monetaria como instrumento para determinar el nivel de actividad y precios).

Dicho de otra manera, en una economía con mercados financieros globalizados y alta movilidad del capital especulativo, las autoridades económicas deben optar entre i) reemplazar el ancla cambiaria por un ancla monetaria, al costo que sea necesario en descontrol de la oferta de deuda pública causado por el alza de la tasa de interés sobre los títulos públicos, desaliento de la inversión privada y del nivel de acti-

\footnotetext{
${ }^{21}$ Lane y Poloz (1992) muestran que la demanda de moneda agregada en los países miembros del Grupo de los 7 tiende a ser más estable que la demanda de cada moneda por separado.
}

vidad y mayor fragilidad del sistema bancario; o bien ii) mantener el tipo de cambio nominal, al costo que sea necesario en atraso cambiario y pérdida consiguiente de recaudación tributaria por operaciones de comercio exterior, en aumento del déficit comercial y en variaciones del acervo de reservas internacionales. Así la 'trinidad imposible' (cambio nominal fijo, movimiento de capitales y autonomía monetaria) puede ser vista como una manifestación de la pérdida de autonomía de las autoridades internas resultante del proceso de globalización financiera.

En sexto lugar, como las inestabilidades cambiarías europeas vienen mostrando desde la crisis de septiembre de 1992 - y como las recientes experiencias mexicana y argentina se encargaron de recordar-, los mercados financieros globales muestran cada vez menos tolerancia con políticas internas que signifiquen atrasos cambiarios significativos o permanentes. Después de la crisis mexicana de diciembre de 1994, el comportamiento de los mercados ha reducido enormemente el margen de maniobra de las autoridades económicas nacionales para manejar el tipo de cambio nominal como ancla del sistema de precios. El casó de México es elocuente, ya que desde entonces debió aceptar la flotación de su moneda. El caso de Argentina supone un fuerte compromiso de no devaluar; sin embargo, el reciente anuncio de una tasa de desempleo de $18.6 \%$ (promedio nacional) puede ser interpretado como un indicador del precio político y social que se debe pagar por insistir en un curso de acción autónomo. El caso de Brasil ilustra una situación intermedia, en la cual el diseño original del Plan Real debió ser alterado - primero adoptando una banda de flotación y más tarde ajusfándola de modo compatible con una devaluación - para satisfacer las expectativas del mercado.

En séptimo lugar, la disciplina impuesta por el funcionamiento de mercados financieros globales alcanza hasta el manejo de metas cuantitativas por parte de las autoridades monetarias. Así por ejemplo, en una economía con tasas de inflación y de crecimiento de la base monetaria iguales a 50\% anual, el anuncio del banco central de que pretende reducir la expansión de la moneda de alta potencia a sólo $48 \%$ anual sería probablemente visto por los mercados como falta de voluntad política para enfrentar el problema. A su vez, si el anuncio fuera que la creación primaria de moneda no excederá el $0 \%$ anual, los mercados probablemente tampoco lo considerarían un anuncio serio (o podrían interpretarlo como información compatible con un futuro congelamiento de precios). 
Por último, en la medida en que numerosas y creativas innovaciones financieras permiten que los agentes del sector privado se puedan proteger contra eventuales cambios en el curso de la política monetaria o cambiaria, la eficiencia de estas últimas tiende a verse disminuida. Esta sería una variante de la ley de Goodhart, cuya versión original establecía que, cuando los agentes económicos descubren la existencia de una relación estable (entre dos o más variables), ésta se quiebra (pues pasa a ser sobreexplotada). En el presente caso, cuando los agentes aprenden a usar los instrumentos surgidos de la desregulación bancaria promovida por el Banco Central, la eficiencia de la política monetaria a cargo de este último tiende a quedar neutralizada (pues los agentes tienen un incentivo para cubrirse contra las iniciativas del Banco Central mediante el adecuado manejo de los referidos instrumentos).

\section{El caso de las autoridades fiscales}

Cuando se trata de ilustrar el efecto restrictivo de la apertura comercial sobre el campo de maniobra de las autoridades fiscales, el primer caso que viene a la mente es el conocido ejemplo del multiplicador del gasto público. En el contexto de políticas keynesianas, destinadas a compensar insuficiencias (o excesos) de demanda del sector privado, la trayectoria de la demanda agregada de una economía cerrada $(\mathrm{D}=\mathrm{Ad}=$ $\mathrm{Cd}+\mathrm{Gd}+\mathrm{Id}$ ) puede ser estabilizada mediante una variación compensatoria en el nivel del gasto público o de la tributación. Sin embargo, a medida que la economía se internacionaliza, la demanda agregada se hace cada vez más sensible a las variaciones en los factores determinantes de la balanza comercial $(\mathrm{D}=\mathrm{Ad}+\mathrm{X}$ $=\mathrm{A}+\mathrm{X}-\mathrm{M}=\mathrm{C}+\mathrm{G}+\mathrm{I}+\mathrm{X}-\mathrm{M})$, con lo que el multiplicador del gasto público disminuye a causa de las 'filtraciones' originadas en la demanda de importaciones.

El conocido argumento anterior resulta de comparar una misma economía en circunstancias de autarquía y en las de apertura, y permite mostrar la pérdida de grados de libertad que experimenta el gobierno central del Estado-nación cuando la economía interna se abre al comercio exterior. ${ }^{22}$ Esto no es suficiente, sin embargo, para mostrar los efectos de la globalización de la economía mundial sobre el grado de autonomía de las autoridades nacionales en el diseño e implementación de sus propias metas de política fiscal. La razón, ya adelantada, es que globalización y apertura no son sinónimos.

\footnotetext{
${ }^{22}$ Se puede obtener una estimación de esta pérdida calculando el aumento necesario en el déficit público para alcanzar un predeterminado grado de expansión en el nivel de actividad o empleo, respecto a lo requerido en una situación de autarquía.
}

Las complejidades fiscales que resultan de la globalización emergen cuando se examina la tributación sobre las utilidades de las empresas transnacionales que establecen administraciones impositivas concurrentes; esto es, las dificultades surgen con la asignación de los derechos de recaudación de impuestos que hacen los diferentes Estados-nación en cuyos territorios las operaciones tienen lugar o donde la casa matriz tiene su sede.

Lo anterior está vinculado al cambio estructural que la globalización viene provocando en el patrón del comercio internacional y de las inversiones extranjeras directas (gráfico 2).

La región A corresponde a las operaciones de comercio exterior a que nos acostumbró la lógica de

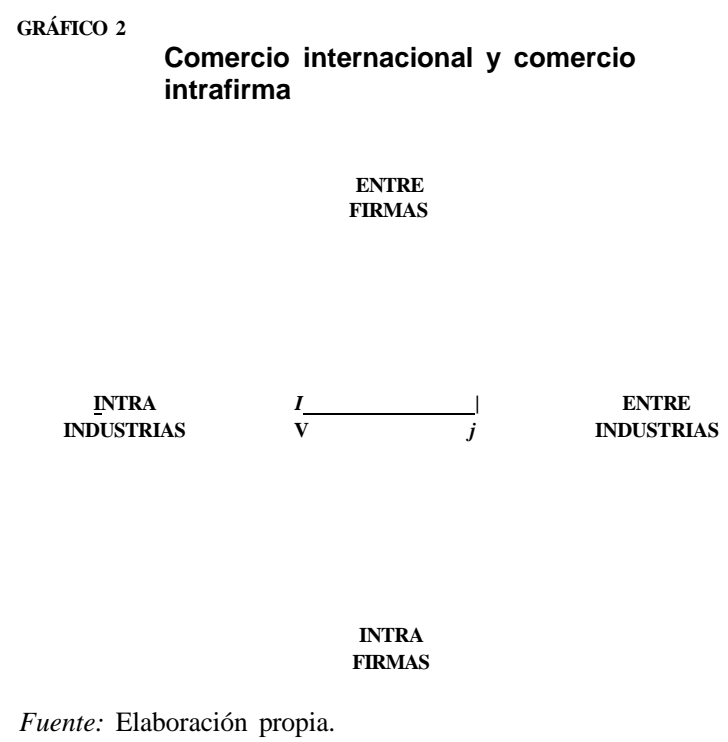


Heckscher-Ohlin. En ella, cada país exporta (importa) el bien que usa intensivamente el factor de producción del cual está mejor (peor) dotado, lo que a su vez da origen a la noción convencional de que el comercio se realiza entre empresas sin vínculos (principio de $a t$ arm's length) de dos países que compran y venden productos de diferentes industrias (digamos, textiles de Inglaterra y vino de Portugal). Este tradicional patrón de comercio internacional entre empresas y entre industrias puede ser interpretado como típico de la fase primitiva del proceso de internacionalización de la economía mundial (aunque es preciso reconocer que aún mantiene vigencia en numerosos productos).

La región $\mathrm{C}$ corresponde a lo que hay de más típico en la actual fase del proceso de globalización. Destacan aquí las operaciones de comercio e inversión intrafirma e intraindustria, efectuadas por empresas transnacionales que operan guiadas por una lógica global y que se apoyan en un esquema organizacional flexible del tipo descrito anteriormente.

Entre los múltiples problemas que las autoridades fiscales deberán enfrentar a medida que las operaciones a través de fronteras (de comercio, inversión y financieras) tiendan a concentrarse en la región $\mathrm{C}$, mencionaremos algunos.

En primer lugar, los arreglos institucionales para el control de flujos a través de fronteras - esto es, las aduanas nacionales - son heredados de los tiempos en que las relaciones económicas internacionales eran típicas de la región A. Por este motivo están básicamente diseñados para lidiar con el comercio de mercaderías pasibles de control físico (toneladas de carne o azúcar, cajas o hectolitros de vino, centenas de neumáticos, número de camiones o automóviles), y realizado por empresas no vinculadas entre sí y que por lo tanto usan precios de mercado para determinar créditos y débitos. Por el contrario, el tipo de transacciones característico de la región $\mathrm{C}$ supone corrientes de comercio que tienen un creciente contenido de servicios, información y conocimientos. En cierta manera, puede decirse que son flujos cada vez más 'conceptuales' y, por lo tanto, su carácter crecientemente intangible tiende a dificultar las valoraciones monetarias que cada país desea realizar con fines arancelarios e impositivos en general.

En segundo lugar, y vinculado al punto anterior, ocurre que los agentes que intervienen en las actividades de la región $\mathrm{C}$ tienden a ser miembros de jerarquías transnacionales para las que lo esencial del comercio internacional es mover bienes y servicios -incluyendo los de tipo financiero_ - al interior de la corporación. El hecho de que esto último involucre mover dichos bienes y servicios entre diversos países es porque hacerlo de esta manera resulta más conveniente para los intereses y estrategia global de la organización. Ocurre que en las operaciones de compraventa interna (entre diferentes subsidiarias, filiales y casa matriz) los 'precios de transferencia' utilizados permiten conocidos manejos cuyas consecuencias tributarias y cambiarías pueden no ser triviales. La importancia del tema para las administraciones tributarias y los bancos centrales de países como el Brasil es considerable, ya que desde el ángulo del comercio de servicios (contabilidad, crédito y finanzas, investigación y desarrollo, capacitación de personal, etc.) y de otros valiosos intangibles (patentes, fórmulas, procesos, diseños, derechos de autor, marcas o nombres comerciales, modelos, franquicias, licencias, datos técnicos, listas de clientes, etc.) es mucho lo que las autoridades fiscales han de hacer para no perder grados de libertad - bajo la forma de pérdida de recaudación - debido al proceso de globalización.

En tercer lugar, es preciso estar atentos al hecho de que el incentivo de las empresas transnacionales para manipular 'precios de transferencia' no se limita al de optimizar su plan tributario global (transfiriendo utilidades desde países con elevada presión impositiva hacia otros con menor carga tributaria). El mismo procedimiento se puede utilizar, entre otras cosas, para repatriar utilidades en exceso de lo permitido por las normativas locales, reducir riesgo en operaciones de cambio, capitalizar activos, evitar controles de precios, responder a cargos de dumping o de otras actividades monopólicas, neutralizar demandas salariales, subvencionar una subsidiaria 'naciente', penetrar nuevos mercados o aumentar la participación en mercados tradicionales. Como puede verse, no son pocos los canales por los que la globalización de la economía puede afectar adversamente, mediante precios de transferencia, los intereses del fisco nacional.

En cuarto lugar, cabe observar el lado financiero de las economías nacionales para ver su creciente interacción con los dinámicos mercados financieros internacionales. En tal contexto, se puede apreciar que los 'bancos globales' —como el Chase Manhattan, el Chemical Bank y otros - representan una modalidad muy especial de jerarquías corporativas transnacionales, por los tipos de 'productos' con que operan y por el tipo de control a que están sujetos en cada país (usualmente bajo la égida del Banco Central). Sin embargo, no se debe desconocer que tales tipos de bancos $-y$ otros agentes financieros que llevan a cabo 
operaciones financieras internacionales - tienen los mismos incentivos (y tal vez más facilidades) que las empresas transnacionales del lado real de la economía para manipular sus respectivos 'precios de transferencia'. Dada la magnitud de los volúmenes manejados por estas instituciones (en operaciones de protección (hedge), especulación y arbitraje intrafirma a través de fronteras), aun pequeñas diferencias en los precios utilizados pueden representar cuantiosas pérdidas para el fisco.

En quinto lugar, la creciente importancia de las empresas transnacionales se manifiesta cada vez más en el volumen de inversión extranjera directa y de comercio que generan (dentro de la región $\mathrm{C}$ del gráfico 2). Cabe imaginar que dichas actividades tienen importantes contrapartidas financieras (como la creación o destrucción de activos financieros vinculados a los pagos de exportaciones e importaciones, operaciones de protección para reducir riesgos cambiarios, operaciones en los mercados de divisas para efectuar remesas de utilidades o repatriación de capital, etc.). Dada la importancia cuantitativa de dichas operaciones en el conjunto de las operaciones externas, el nivel medio de las reservas internacionales y el volumen de operaciones en el mercado de divisas, es evidente la necesidad de que los bancos centrales sigan de cerca lo que las empresas transnacionales puedan estar haciendo (o venir a hacer), de manera coordinada con 'bancos globales', en materia de precios de transferencia.

En sexto lugar, existen diversas áreas nichos en las que se viene dando una activa interacción entre los lados real y financiero de las empresas transnacionales. Así por ejemplo, éstas poseen en todo momento activos y pasivos líquidos denominados en diversas monedas y situados en numerosos países. Por lo tanto, los movimientos de divisas generados por las técnicas de manejo de los flujos de caja de estas empresas — dado el volumen que representan y la asiduidad con que tienen lugar - les permite transferir cifras enormes aún con la adopción de pequeños diferenciales de tasas de interés (spreads). Otro ejemplo de estas áreas nichos se encuentra en la industria de los llamados 'seguros cautivos', esto es, empresas de seguros que son subsidiarias de una empresa transnacional del sector no financiero (por ejemplo, una fábrica de automóviles) o controladas por ella. La motivación para crear estas empresas aseguradoras 'cautivas' puede inferirse del hecho de que en 1991 el $75 \%$ de ellas se hallaba en paraísos fiscales extraterritoriales. De esto también se infiere la pérdida de autonomía para las autoridades fiscales que significan ésta y otras modalidades de operación de las empresas transnacionales.

En séptimo lugar, y por último, la carga tributaria de las empresas transnacionales en un país también puede ser alterada i) sustituyendo corrientes de comercio por corrientes de inversión extranjera directa (cuyas utilidades suelen estar exentas de gravámenes durante algunos años); ii) reorientando la producción hacia la exportación de bienes manufacturados (con lo cual el mecanismo de devolución del impuesto al valor agregado permite reducir la contribución neta de sus operaciones al Tesoro), y iii) modificando la composición de sus exportaciones en favor de los servicios (con lo cual los administradores tributarios quedan prácticamente imposibilitados de ejercer adecuada fiscalización).

Los ejemplos anteriores apenas tocan algunas de las múltiples y complejas formas en que el comportamiento y las estrategias del principal agente del proceso de globalización - esto es, las empresas transnacionales del lado real o financiero- pueden reducir la autonomía de las autoridades fiscales nacionales. Por cierto, existen muchos otros canales que este ensayo no explora y que no se vinculan directa ni necesariamente a estos agentes económicos.

Sólo para ilustrar esto último, retomemos el caso de pequeñas economías abiertas a los movimientos de capitales de corto plazo y que mantienen fijo el tipo de cambio nominal como parte de un programa antiinflacionario. Como cabe imaginar, una abundante disponibilidad de financiamiento externo tiene como contrapartida el potencial efecto adverso de la excesiva monetización sobre la meta inflacionaria. Esto fuerza la intervención del Banco Central para fines de esterilización, con el consecuente aumento en la oferta de títulos internos y en la tasa de interés.

Lo anterior tiene dos consecuencias al menos: i) por una parte, refuerza el sistema de incentivos que atrae la entrada neta de capitales (lo que permite anticipar que en el futuro, a igualdad de otras condiciones, habrá nuevas intervenciones esterilizadas con aumentos de la tasa de interés para asegurar que el mercado continúe absorbiendo cada vez más valores internos); ii) por otra, tiende a hacer más frágiles las cuentas públicas, debido al correspondiente aumento del servicio de la deuda pública. Como lo anticipaba Calvo (1991), esta combinación de efectos promueve la pérdida de confianza de los agentes económicos en la sustentabilidad del programa antiinflacionario, y tiene un efecto más acentuado cuanto mayor sea la entrada neta de capitales de corto plazo. 


\section{VI}

\section{Comentarios finales}

La globalización de la economía mundial es un proceso en marcha cuyas consecuencias de largo plazo en diferentes áreas hoy sólo es posible atisbar.

Sin embargo, ya está claro que dicho proceso plantea extraordinarios desafíos a los responsables de la estabilidad del sistema financiero y de la conducción de las políticas monetaria y cambiaria. De igual modo, los administradores tributarios y demás encargados del resto de la política fiscal enfrentan crecientes dificultades para concebir y aplicar políticas capaces de neutralizar los efectos adversos de la globalización sobre la autonomía de las autoridades económicas.

El fenómeno de la globalización efectivamente limita el margen de maniobra de los gobiernos nacionales. Sin embargo, la fuerza disciplinadora de la competencia internacional que subyace al menos en buena parte del proceso puede llegar a tener importantes efectos benéficos sobre el curso futuro de las políticas públicas en los países de la región. Así, cuando se hable de 'pérdida de autonomía' habrá que mirar con cuidado para ver si acaso no se trata apenas de una bienvenida 'reducción en el grado de arbitrariedad' con que a veces se manejan las políticas públicas. Cabe preguntarse, por ejemplo, si la creciente falta de tolerancia de los mercados financieros internacionales - a manejos arbitrarios del tipo de cambio, o también a déficit públicos elevados y persistentesafecta la autonomía de las autoridades internas (puesto que aumenta la importancia de las restricciones que los gobiernos deben obedecer), o si más bien es una reacción benéfica que evita males mayores a futuro (como la acumulación de grandes rezagos cambiarios que, a la hora de la inevitable devaluación, originan traumas financieros con considerables efectos negativos en la esfera real de la economía).

En todo caso, el Estado-nación que conocemos deberá permanecer aún por mucho tiempo como unidad organizadora básica de la vida política, económica, social y cultural de nuestros pueblos. ${ }^{23}$ La globalización le trae nuevos y complejos desafíos, pero también enormes oportunidades. Las autoridades económicas nacionales disponen de poderosos instrumentos para manejar los primeros y deben armarse de equivalente entusiasmo - a la vez que desarrollar enorme esfuerzo - para aprovechar las segundas. Entre las muchas opciones abiertas a los gobiernos nacionales - no consideradas aquí por razones de extensiónconviene tener presente la propuesta de la CEPAL (1994) en favor del regionalismo abierto. Como muestra Stallings (1995), los gobiernos nacionales ya vienen dando importantes respuestas regionales a los desafíos globales. Queda abierta la pregunta sobre cuál de los grandes esquemas de integración a través de los que se expresan dichas respuestas — sea en la tradición del Tratado de Roma (donde el liderazgo del proceso recae sobre jerarquías gubernamentales, como en el MERCO SUR), sea siguiendo la experiencia china (en que el liderazgo es ejercido por agentes privados a través de los mercados) - es menos dañino para la futura auto* nomía del Estado-nación. ${ }^{24}$

\footnotetext{
${ }^{23}$ La tesis de la obsolescencia del Estado-nación y surgimiento del Estado-región tiene uno de sus más destacados expositores en Ohmac (1993 y 1995).
}

\footnotetext{
${ }^{24}$ Sobre la experiencia de China, véase Jones, King y Klein, 1992.
} 


\section{Bibliografía}

Alcorta, L. (1993): Are economies of scope replacing economies of scale?: Implications for developing countries, documento presentado en la primera Conferencia INTECH, Maastricht, Países Bajos, 21 al 23 de junio, mimeo.

Banco Mundial (1993): The East Asian Miracle: Economic Growth and Public Policy, Washington, D.C.

Baily, M.N. y H. Gersbach (1995): Efficiency in Manufacturing and the Need for Global Competition, Brooking Papers on Economic Activity: Microeconomics.

Baumann, R. (comp.) (1995): Uma visão econômica da globalização, O Brasil e a economia global, Rio de Janeiro, Editora Campus Ltda.

Bierksteker, T. J. (1995): The triumph of liberal economic ideas in the developing world, B. Stallings (éd.), Global Change, Regional Response, The New International Context of Development, cap. 6, Londres, Cambridge University Press.

Calvo, G. (1991): The perils of sterilization, IMF Staff Papers, $\mathrm{N}^{\circ}$ 38, Washington D.C, Fondo Monetario Internacional (FMI).

CEPAL (1994): El regionalismo abierto en América Latina y el Caribe, LC/L. 808 (CEF.19/3), Santiago de Chile.

Crockett, A. (1994): A BIS view of the global banking issues of the 1990's, BIS Review, N 75, Basilea, Banco de Pagos Internacionales (BPI), abril.

Di Filippo, A. (1995): La integración de la economía mundial: los procesos de globalización y regionalización, documento de apoyo al Taller Interamericano sobre Estadísticas del Comercio Regional y las Finanzas Externas de América Latina, Santiago de Chile, Centro Interamericano de Enseñanza de Estadística (CIENES), junio-agosto, mimeo.

Dollar, D. (1993): Technological differences as a source of comparative advantage, The American Economic Review, vol. 83, $\mathrm{N}^{\circ} 2$, Los Angeles, Calif., American Economic Association, mayo.

Dunning, J.H. (1993): Multinational Enterprises and the Global Economy, Reading, Mass., Addison-Wesley.

Fischer, S. (1987): Economic growth and economic policy, Symposium on Growth-Oriented Adjustment Programs, Washington, D.C, Banco Mundial/Fondo Monetario Internacional (FMI), febrero.

FMI (1995): World Economic Outlook, Washington, D.C, mayo.

Freeman, C. (1994): The economics of technical change, Cambridge Journal of Economics, vol. 18, No. 5, Londres, Academic Press, octubre.

Gerschenkron, A. (1966): Economic Backwardness in Historical Perspective, Cambridge, Mass., Harvard University Press.

Gray, P. H. (1994): The efficiency of financial intermediation and international competitiveness, Weltwirtschaftliches Archiv. Review of World Economics, Band 130, Heft 4, Tubingen, Alemania, Institut für Weltwirtschaft Kiel.

Griffith-Jones, S. y B. Stallings (1995): New global financial trends: implications for development, B. Stallings (éd.): Global Change, Regional Response. The New International Context of Development, Londres, Cambridge University Press.

Harberger, A.C (ed.)(1984): Economic policy and economic growth, World Economic Growth, San Francisco, Calif., Institute for Contemporary Studies.

(1995): Monetary and fiscal policy for equitable economic growth, Conference on Income Distribution and Sustainable Growth, Washington, D.C, FMI, junio.

Hummels, D. y J. Levinsohn (1993): Product differentiation as a source of comparative advantage?, The American Economic Review, vol. 83, $\mathrm{N}^{\circ} 2$, Los Angeles, Calif., American Economic Association.
Jones, R., R. King y M. Klein (1992): The Chinese Economic Area: Economic Integration without a Free Trade Agreement, Working Papers, $\mathrm{N}^{\circ} 153$, Paris, Economic Department, Organización de Cooperación y Desarrollo Económicos (OCDE).

Krugman, P. (1995): Dutch tulips and emerging markets, Foreign Affairs, vol. 74, $\mathrm{N}^{\circ}$ 4, Nueva York, Council on Foreign Relations, Inc., julio-agosto.

Lamfalussy, A. (1995): Financial innovation and market stability, BIS Review, $\mathrm{N}^{\circ} 18$, Basilea, Suiza, BPI, febrero.

Lane, T. y S. Poloz (1992): Currency Substitution and Cross-Border Monetary Aggregation: Evidence from the G-7, IMF Working Paper, No.81, Washington, D.C, FMI, octubre.

Learner, E. E. (1993): Factor-Supply Differences as a Source of comparative advantage, The American Economic Review, vol. 83, N ${ }^{\circ}$ 2, Los Angeles, Calif., American Economic Association, mayo.

Little, I.M., R.N. Cooper, W.M. Corden y S. Rajapatirana (1994): Auge, crisis y ajuste: la experiencia macroeconómica de los países en desarrollo, 1970-90, Washington, D.C, Banco Mundial/Oxford University Press.

Metcalfe, J.S. (1994): Evolutionary economics and technology policy, The Economic Journal, vol. 104, $\mathrm{N}^{\circ} 425$, Oxford, Reino Unido, Blackwell Publishers, julio.

Milgrom, P. y J. Roberts (1990): The economics of modern manufacturing: technology, strategy and organization, The American Economic Review, vol. 80, No 3, Los Angeles, Calif., American Economic Association, junio.

Nelson, R. (1995): Recent evolutionary theorizing about economic change, Journal of Economic Literature, vol. XXXIII, $\mathrm{N}^{\circ} 1$, Menasha, Wis., The American Economic Association, marzo.

Nelson, R. y S. Winter (1982): Evolutionary Theory of Economic Change, Cambridge, Mass., The Belknap Press of Harvard University Press.

North, D. (1990): Institutions, Institutional Change, and Economic Performance, Londres, Cambridge University Press.

O'Brien, R. (1992): Global financial integration: The end of geography, Chatham House Papers, $\mathrm{N}^{\circ} 7$, Londres, Royal Institute of International Affairs.

Ohmac, Kenichi (1993): The rise of the Region State, en Foreign Affairs, Boston, primavera.

(1995): Putting global logic first, en Harvard Business Review, enero-febrero.

Oman, C (1994): Globalisation and Régionalisation: The Challenge for Developing Countries, París, Centro de Desarrollo, OCDE.

Parente, S.L. y B.C. Prescott (1991): Technology adoption and growth, Research Department, Staff Report, $\mathrm{N}^{\circ}$ 136, Minneapolis, Federal Reserve Bank of Minneapolis, febrero.

Porter, M. E. (1991): La ventaja competitiva de las naciones, Buenos Aires, J. Vergara Editor,

(1992): Capital disadvantage: America's failing investment system, Harvard Business Review, Cambridge, Mass., septiembre- octubre.

Prowse, S. (1994): Corporate governance in an international perspective: A survey of corporate control mechanisms among large firms in the United States, the United Kingdom, Japan and Germany, BIS Economic Papers, $\mathrm{N}^{\circ}$ 41, Basilea, Suiza, BPI, julio.

Rosenberg, N. (1994): Exploring the Black Box: Technology, Economics, and History, Londres, Cambridge University Press.

Stallings, B. (1995): Introduction: global change, regional response, en B.Stallings (éd.), Global Change, Regional Response: The New International Context of Development, Cambridge, Mass., Cambridge University Press. 
Storper, M. (1995): Territories, flows and hierarchies in the global economy, Aussenvirtschaft, $\mathrm{N}^{\circ} 50$, Zurich, Riiegger.

Stoneman, P. Y P. Diederen (1994): Technology diffusion and public policy, The Economic Journal, vol. 104, $\mathrm{N}^{\circ} 425$, Oxford, Reino Unido, Blackwell Publishers, julio.

Tybout, J.R. (1993): Internal returns to scale as a source of comparative advantage: the evidence, The American Economic Review, vol. 83, № 2, Los Angeles, Calif., American Economic Association, mayo.
UNCTAD (Conferencia de las Naciones Unidas sobre Comercio y Desarrollo) (1993): World Investment Report 1993, Transnational Corporations and Integrated International Production, Publicación de las Naciones Unidas, $\mathrm{N}^{\circ}$, de venta E.93.II.A.14.

Williamson, O.E. y S.G. Winter (1991): The Nature of the Firm: Origins, Evolution, and Development, Oxford, Reino Unido, Oxford University Press.

Williamson, J. (1993): Democracy and the Washington Consensus, World Development, vol. 21, $\mathrm{N}^{\circ}$ 8, Oxford, Reino Unido, Pergamon Press Ltd. 